\title{
The concepts of Theological Principles in morality literatures and in the Holy Bible
}

\section{இறைநெறிக் கொள்கைகளில், அறஇலக்கியமும், திருவிவிலியமும்}

\section{A. Bavliance a, *}

a Department of Tamil, Alagappa Government Arts College, Sivaganga-630003, Tamil Nadu, India.

*Corresponding author Email: anthonybavliance8421@gmail.com

DOI: https://doi.org/10.34256/ijmrd2136

Received: 30-06-2021; Revised: 11-08-2021; Accepted: 11-08-2021; Published: 15-08-2021

Abstract: In the praise of the Lord who creates the world, the scriptures of the eighteen following books of the Sangamamaruviya period, and the Holy Book of Christianity, the Holy Book of Christianity, are of the Lord. This article explains that humanity is cultivated by the praise of sovereignty, removes evils, and directs the way of good ness. In the article, the article explains the importance of appreciating the nature of God and the system of worship of God, the benefits and the virtues of the sovereignty, that the world is the creation of the Lord and that the atom will not move without him.

Keywords: Sangamamaruviya period, Holy Book of Christianity, God, Sovereignty

Language: Tamil

முன்னுரை

ஆலயம் தொழுவது சாலவும் நன்று

இறைமை என்பது சமயங்கள் போற்றும் அறநெறி (Venkatasamy Nattar, 2018). மனிதனை பண்படுத்தும் உன்னதக் கருவியாகும். மனிதனின் அறிவுக்கு எட்டாத அருஉருவமான நிலைப்பாடு. தமிழ் இலக்கியங்களில் அறச்சிந்தனைகளை முழுமையாக வழங்கும் பதினெண்கீழ்க்கணக்கு நூல்களிலும் கிறிஸ்தவர்களின் புனித நூலான திருவிவிலியத்திலும் இறைநெறிக் கொள்கைகள் சிறந்து விளங்குகிறது என்பது வெள்ளிடை மலை.

\section{அற இலக்கியங்களில் இறைநெறிக் கொள்கைகள்}

உலகப் பொதுமறையாம் திருக்குறளில் வள்ளுவப் பெருந்தகை தம் முதல் குறட்பாவில்

அகர முதல எழுத்தெல்லாம் ஆதி

பகவன் முதற்றே உலகு.

எழுத்துகள் அகரத்தை முதன்மையாகக் கொண்டு இயங்குகிறது. இவ்வுலகம் எல்லாவற்றிற்கும் ஆதியாக இருக்கும் இறைவனை முதன்மையாகக் கொண்டு இயங்குகிறது என்கிறார் (Parimelalhagar, 2002).

புறவிப் பெருங்கடல் நீந்துவர் நீந்தார்

இறைவன் அடிசேரா தார். 
இப்பாடலில் மனிதப்பிறவி என்னும் பெருங்கடலைக் கடக்க வேண்டுமானால் இறைவனின் அடியை பற்றிக் கொள்ள வேண்டும். அப்படி பற்றிப் பிடிக்காதவர் பிறவிப் பெருங்கடலை நீந்திக் கரைசேர முடியாது என்கிறார் (Parimelalhagar, 2002).

\section{நான்மடிக்கடிகை}

மதிமன்னும் மாயவன் வாள் முகம் ஒக்கும்

கதிர் சேர்ந்த சக்கரம் ஒக்கும்

முதுநீர்ப் பழநத்து தாமரைத் தாளின்

ஏதிர்மலர் மற்றவன்கண் ஒக்கும்; பூவைப்

புதுமலர் ஓக்கும் நிறம்.

திங்கள் அழிதலில்லாத திருமாலின் ஒளியையும் ஒளி சிறந்த ஞாயிறு அவனது சக்கரப் படையையும் நீர்நிலைகளில் முளைத்துள்ள செந்தாமரைப்பூ அவன் கண்களையும் காயாமரத்தின் பூ அவனது திருமேனியையும் ஒத்திருக்கும் என்று இறைவனின் சாயலில் அனைத்தும் இருக்கிறது என்பதைப் போற்றிப் பாடுகிறது (Jagathrakshakan, 2021).

\section{இன்னா நாற்பது}

முக்கட் பகவ னடிதொழா தார்க்கிண்ணா

பொற்பனை வெள்ளையை யுள்ளா தொழுகின்னா

சக்கரத்தானை மறப்பின்னா வாங்கின்னா

சத்தியான் றாடொழா தார்க்கு.

மூன்று கண்களையுடைய சிவபெருமானையும் அழகிய பனைக்கொடியை உடைய பலராமனையும் சக்கரப் படையையுடைய திருமாலையும் வேற்படையையுடைய முருகப்பெருமானையும் வணங்காதவர்களுக்கு துன்பம் உண்டாகும் என்பதைத் தெளிவுப்படுத்துகிறது (Gowmareeswari, 2015).

\section{இனியவை நாற்பது}

கண்மூன் றுடையாண்தாள் சேர்தல் கடிதினிதே

தொண்மான் துழாய்மாலை யானை தொழலினிதே

எனும் பாடலில் மூன்று கண்களையுடைய சிவபெருமானது திருவடிகளை அடைதல் மிக எளிது. பழமையாகிய மாட்சி பெற்ற திருத்துழாய் மாலையை அணிந்த திருமாலை கைதொழுதல் இனிது.

நூன்கு முகங்களையுடைய பிரம்ம தேவனை அவன் முன்னிருந்து விரும்பி வாழ்த்துவது இனிது என்று இறைவனை வணங்கி வாழ்த்துபவர்களுக்கு வாழ்வு இனிமையாகும் என்று பாடுகிறது (Gowmareeswari, 2015).

\section{ஆசாரக்கோவை}

நாளந்திக் கோறின்றி கண்கழீஇத் தெய்வத்தை

தானையும் மாற்றாற் தொழுதெழுக வல்கந்தி 
நின்று தொழுதல் பழி.

விடியற்காலையில் ஒருகோலை எடுத்து பல் துடைத்து கண்களைக் கழுவித் தான் வணங்கும் கடவுளை அறியும் நெறியால் தொழுக, பின் ஒரு செயலைத் தொடங்க வேண்டும். மாலைப் பொழுதின்கண் தான் வணங்கும் கடவுளை நின்று கொண்டு வணங்குதல் குற்றமாகும். இருந்து வணங்குதல் சிறப்பு என்று இயம்புகிறது (Gowmareeswari, 2015).

\section{முதுமொழிக்காஞ்சி}

ஆர்கலி உலகத்து மக்கட் கெல்லாம்

புகழ்வெய் யோர்க்குப் புத்தேணா டெளிது.

கடல் சூழ்ந்த இவ்வுலகத்து மக்கள் எல்லோருள்ளும் புகழப்படும் அறச்செயல்களைச் செய்ய விரும்பினார்க்கு தேவர்கள் வாழும் விண்ணுலகம் பெறுவது எளிதென்று இறைவன் விரும்புவது 'அறம'; என்று பாடுகிறது (Gowmareeswari, 2015).

\section{இறைநெறிக் கொள்கைகளில் திருவிவிலியம்}

ஆதியில் உலகைப் படைத்த இறைவன் படைப்பின் சிகரமாக மனிதனைப் படைத்தார். ஆனால் மனிதன் தீமைகளால் பாவக்குழியில் விழுந்து அழிவதை விரும்பாமல் தன் ஒரே மகனாகிய இயேசு கிறிஸ்துவை மனிதனாகப் பிறப்பெடுக்க வைத்தார். அவர்தம் சிலுவைப் பாடுகளால் இவ்வுலகை மீட்டெடுக்க தன்னையே பலியாக்கினார். தந்தையாம் இறைவனின் விருப்பத்தை நிறைவேற்றினார் என்று பொதுநலம் பாடும் திருவிவிலியம் உரைக்கிறது (Holy Bible Thiruviviliam, 1995).

திருப்பாடல்: அல்லேலூயா! தூயகத்தில் இறைவனை போற்றுங்கள். வலிமைமிகு விண்விரிவில் அவரைப் போற்றுங்கள். அவர்தம் வல்ல செயல்களுக்காக அவரைப் போற்றுங்கள். அவர்தம் எல்லையில்லா மாண்பினைக் குறித்து அவரைப் போற்றுங்கள் என்றும் எக்காளம் முழங்கியும் வீணையுடன் யாழ் மீட்டியும் மத்தளம் கொட்டியும் குழலினை ஊதியும் சிலம்பிடும் சதங்கையுடன் கலீர் எனும் தாளத்துடனும் அவரைப் போற்றுங்கள். அனைத்து உயிர்களே ஆண்டவரைப் புகழ்ந்திடுங்கள் என்று இறைவனைப் போற்றும் முறைமைகளை மிகத் தெளிவாக எடுத்துரைக்கிறது.

லூக்கா நற்செய்தியில் ஆறாம் மாதத்தில் கபிரியேல் எனும் வான தூதர் கலிலேயாவில் நாசரேத் ஊரில் தாவீதின் குடும்பத்தில் யோசேப்பு என்பவருக்கு மண ஒப்பந்தமான மரியாவுக்குத் தோன்றி "அருள்மிகுப் பெற்றவளே வாழ்க, ஆண்டவர் உம்முடனே" என்றார். இவ்வாழ்த்து எத்தகையதோ என்று அஞ்சிய மரியாளுக்கு "மரியா அஞ்ச வேண்டாம்; கடவுளின் அருளைக் கண்டுள்ளீர்; இதோ கருவுற்று ஒரு மகனைப் பெற்றெடுப்பீர், அவர் கடவுளின் மகன் எனப்படுவார். அவருடைய தந்தை தாவீதின் அரியணையை கடவுள் அவருக்கு அளிப்பார்" என்று உரைத்தார்.

மரியாவின் பாடல் ஆண்டவரை எனது உள்ளம் போற்றி பெருமைபடுத்தும் என் மீட்பராம் கடவுளை நினைந்நு பேருவகை கொள்கிறது. ஏனெனில் அவர்தம் அடிமையின் தாழ்நிலையை கண்ணோக்கினார். இதுமுதல் எல்லாத் தலைமுறையினரும் என்னை பேறு பெற்றவர் என்பர். வல்லவராம் கடவுள் எனக்கு அரும்பெருஞ்செயல்கள் புரிந்துள்ளார். தூயவர் என்பது அவரது பெயர். அவருக்கு அஞ்சி நடப்போருக்கு தலைமுறை தலைமுறையாய் இரக்கங்காட்டுகிறார்.

மத்தேயு நற்செய்தியில் இயேசு போதித்துக் கொண்டிருந்தபொழுது, திருச்சட்ட அறிஞர் ஒருவர் எழுந்து, அவரை சோதிக்கும் பொருட்டு போதகரே! நிலைவாழ்வை உரிமையாக்கிக் கொள்ள நான் என்ன செய்ய வேண்டும் எனக் கேட்க, இயேசு மறுமொழியாக 
“உன் முழு இதயத்தோடும் முழு மனத்தோடும் உன் கடவுளாகிய ஆண்டவரிடம் அன்பு கூறுவாயாக உன் மீது அன்பு கூர்வது போல உனக்கு அடுத்திருப்பவர் மீதும் அன்பு கூர்வாயாக என எழுதியுள்ளதே” என்றார். இவ்வாறு இறைவனையும் பிறரையும் முழு மனதோடு அன்பு செய்ய வேண்டுமென்று திருவிவிலியம் வாக்குரைக்கிறது (Holy Bible Thiruviviliam, 1995).

இயேசு தன் சீடர்களுக்கு செபிக்கக் கற்றுக்கொடுத்தபோது தந்தையே உமது பெயர் தூயது எனப் போற்றப்பெறுக, உமது ஆட்சி வருக. உமது திருவுளம் விண்ணுலகில் நிறைவேறுவது போல மண்ணுலகிலும் நிறைவேறுக.

ஏங்கள் அன்றாட உணவை நாள்தோறும் எங்களுக்குத் தாரும். எங்களுக்கு எதிராக குற்றம் செய்வோரை நாங்கள் மன்னிப்பதால் எங்கள் பாவங்களையும் மன்னியும். எங்களை சோதனைக்கு உட்படுத்தாதேயும் தீயோரிடமிருந்து எங்களை விடுவித்தருளும் என்று இறைவனைப் போற்றிப் புகழ்ந்து, துன்பங்களைத் தீர்க்கவும் பாவங்களை மன்னிக்கவும் தீமையிலிருந்து விடுவிக்கவும் செபிக்க கற்றுக்கொடுத்ததை உணர முடிகிறது.

கேளுங்கள் உங்களுக்கு கொடுக்கப்படும்; தட்டுங்கள் திறக்கப்படும்; தேடுங்கள் கண்டடைவீர்கள். ஏனெனில் கேட்போரெல்லாம் பெறுகின்றனர். தேடுவோர் கண்டடைகின்றனர். தட்டுவோர்க்கு திறக்கப்படும். விண்ணகத்தந்தை தம்மிடம் கேட்போருக்கு தூய ஆவியைக் கொடுப்பது எத்தனை உறுதி.

இதன்மூலம் இறைவனிடம் கேட்கும் ஒருவர் பெறுகிறார். தேடுவோர் கண்டடைகிறார், தட்டுவோர்க்குத் திறக்கப்படும் என்ற நம்பிக்கையை உணர்த்துகிறது இவ்விறைவாக்கு.

மத்தேயு நற்செய்தியில் ஆகவே நான் உங்களுக்குச் சொல்கிறேன் உயிர்வாழ எதை உண்பது, எதைக் குடிப்பது, உடலுக்கு எதை உடுத்துவது என்றோ நீங்கள் கவலை கொள்ளாதீர். உணவை விட உயிரும், உடையை விட உடலும், உயர்ந்தது அல்லவா

வானத்துப் பறவைகளைப் பாருங்கள்... கவலை உங்களை ஒரு முழம் கூடக் கூடச் செய்யாது... இன்றைக்கு இருந்து நாளைக்கு மடியும் காட்டுப்புல்லுக்கே கடவுள் இவ்வாறு செய்கிறாராயின் உங்களுக்கு இன்னும் அதிகமாக செய்வாரல்லவா! என்று இறைவன் நம்மைக் கைவிடமாட்டார்; நீ கவலைப்படுவதால் ஓன்றும் ஆகாது; உன்னைக் கடவுள் பார்த்துக் கொள்வார் உனக்குத் தேவையானவற்றை அவர் தருவார் என்று இறைவனின் இயல்பை எடுத்தோதுகிறது.

இயேசு விண்ணரசுப் பற்றிக் கூறும்போது உவமைகள் வாயிலாக பேசினார். கடுகு விதை ஒருவர் கடுகு விதை எடுத்து வயலில் விதைத்தார். அவ்விதை எல்லா விதைகளையும் விட சிறியது. ஆனாலும் அது வளரும்போது மற்றெல்லாச் செடிகளையும் விட பெரியதாகும். வானத்துப் பறவைகள் அதன் கிளைகளில் வந்து தங்கும். விண்ணரசு இக்கடுகு விதைக்கு ஒப்பாகும். புளிப்பு மாவு பெண் ஒருவர் புளிப்பு மாவை எடுத்து மூன்று மரக்கால் மாவில் பிசைந்து வைத்தார். மாவு முழுவதும் புளிப்பேறியது. விண்ணரசு இப்புளிப்பு மாவுக்கு ஒப்பாகும். புதையல் உவமை ஒருவர் நிலத்தில் மறைந்திருந்த புதையலைக் கண்டுபிடிக்கிறார். அவர் அதை மூடிவைத்து விட்டு மகிழ்ச்சியுடன் போய்த் தமக்குள்ள யாவற்றையும் விற்று அந்த நிலத்தை வாங்கிக் கொள்கிறார். விண்ணரசு இப்புதையலுக்கு ஒப்பாகும் (Holy Bible Thiruviviliam, 1995). முத்து உவமை வணிகர் ஒருவர் நல்ல முத்துக்களைத் தேடிச் செல்கிறார். விலை உயர்ந்த ஒரு முத்தைக் கண்டவுடன் அவர் போய் தமக்குள்ள யாவற்றையும் விற்று அதை வாங்கிக் கொள்கிறார். விண்ணரசு அந்நிகழ்ச்சிக்கு ஒப்பாகும். ஆண்டவரே! நீரே என் கடவுள். நூன் உம்மை மேன்மைப்படுத்துவேன். உம் பெயரைப் போற்றுவேன். ஆண்டவரிடம் கொள்ளும் அச்சமே ஞானத்தின் தொடக்கம்.

\section{முடிவுரை}

இவ்வுலகத்தின் இயக்கம் இறைவனால் என்பதை தொன்மையான தமிழ்மொழியின் அற இலக்கியங்களான பதினெண்கீழ்க்கணக்கு நூல்களிலும் கிறிஸ்தவ சமய புனித நூலான 
திருவிவிலியமும் தெற்றென விளக்குகிறது. இறைமை போற்றல் மனிதனை தீமைகளிலிருந்து விலக்கி அறச் சிந்தனைகளை வளர்த்து நன்மைகளை நோக்கி வழிநடத்துகிறது. இறைமை போற்றல் தூய்மையையும் நம்பிக்கையையும் அன்புறவையும் கீழ்ப்படிதலையும் பகிர்வு மனப்பான்மையையும் பிற உயிர்களுக்கு உதவும் பான்மையையும் வளர்க்கிறது. மானுடத்தைப் பண்படுத்தி மன்பதையை அமைதியும் இனிமையும் நிறைந்ததாக வழிநடத்துகிறது என்பதே நாம் உணரும் இறைநெறிக் கொள்கைகளின் உண்மையாகும்.

\section{References}

----, (1995) Holy Bible Thiruviviliam, Tamil Nadu Biblical, Catechetical \& Liturgical Centre, Thindivanam, India.

Gowmareeswari, S., (2015) Pathinenkilkanakku Noolgal Moolam Uraiyum, Saradha Pathippagam, Chennai, India. Jagathrakshakan, S., (2021) Nanmanikkatigai, Pustaka Digital Media, Karnataka, India.

Parimelalhagar, (2002) Thirukural, Saradha Pathippagam, Chennai, India

Venkatasamy Nattar, N.M., (2018) Kondrai Vendhan, Createspace Independent Pub, Chennai, India

\section{Funding}

No funding was received for conducting this study.

\section{Conflict of interest}

The Author has no conflicts of interest to declare that they are relevant to the content of this article.

\section{About the License}

(C) The author 2021. The text of this article is open access and licensed under a Creative Commons Attribution 4.0 International License

\section{Cite this Article}

A. Bavliance, The concepts of Theological Principles in morality literatures and in the Holy Bible, Indian Journal of Multilingual Research and Development, Vol 2, Iss 3 (2021) 47-51. DOI: https://doi.org/10.34256/ijmrd2136 